\title{
Some applications of generalized open sets via operations
}

\author{
Baravan A. Asaad \\ Deptartment of Mathematics, Faculty of Science, University of Zakho, Kurdistan-region, Iraq \\ Received: 28 August 2016, Accepted: 25 November 2016 \\ Published online: 11 January 2017.
}

\begin{abstract}
This paper introduces the concept of an operation on $\tau_{g}$. Using this operation, we define the concept of $g$ - $\gamma$-open sets, and study some of their related notions. Also, we introduce the concept of $g \gamma$-generalized closed sets and then investigate some of its properties. Furthermore, we introduce and investigate $g-\gamma$ - $T_{i}$ spaces $\left(i \in\left\{0, \frac{1}{2}, 1,2\right\}\right)$ and $g$ - $(\gamma, \beta)$-continuous functions by utilizing the operation $\gamma$ on $\tau_{g}$. Finally, some basic properties of functions with $g$ - $\beta$-closed graphs have been obtained.
\end{abstract}

Keywords: $g$ - $\gamma$-open sets, $g \gamma g$-closed sets, $g-\gamma$ - $T_{i}$ spaces $\left(i \in\left\{0, \frac{1}{2}, 1,2\right\}\right), g$ - $(\gamma, \beta)$-continuous functions, $g$ - $\beta$-closed graphs.

\section{Introduction}

Generalized open sets play a very important role in general topology and they are now the research topics of many topologists worldwide. Indeed a significant theme in general topology and real analysis concerns the variously modified forms of continuity, separation axioms compactness, connectedness etc. by utilizing generalized open sets.

Generalized closed ( $g$-closed) sets in a topological space were introduced by Levine [5] in order to extend many of the important properties of closed sets to a larger family. For instance, it was shown that compactness, normality, and completeness in a uniform space are inherited by $g$-closed subsets.

Kasahara [6] defined the concept of an operation on $\tau$ and introduced the concept of $\alpha$-closed graphs of functions. After the work of Kasahara, Jankovic [4] defined the concept of operation-closures of $\alpha$ and investigated function with strongly closed graph. Ogata [8] defined and studied the concept of operation-open sets ( $\gamma$-open sets), and used it to investigate operation-separation axioms and operation-functions.

In recent years, many concepts of operation $\gamma$ in a topological space $(X, \tau)$ have been developed. An, Cuong and Maki [1] developed an operation $\gamma$ on the collection of all preopen subsets of $(X, \tau)$ to introduce the notion of pre- $\gamma$-open sets. Krishnan, Ganster and Balachandran [7] defined and investigated the concept of the mapping $\gamma$ on the collection of all semiopen subsets of $(X, \tau)$, and introduced the notion of semi $\gamma$-open sets and studied some of their properties. Tahiliani [9] developed an operation $\gamma$ on the collection of all $\beta$-open subsets of $(X, \tau)$ to describe the notion of $\beta$ - $\gamma$-open sets and Carpintero, Rajesh and Rosas [2] developed an operation $\gamma$ on the collection of all $b$-open subsets of $(X, \tau)$ to define the notion of $b$ - $\gamma$-open sets.

The aim of this paper is to introduce the concept of an operation $\gamma$ on $\tau_{g}$ and to define the notion of $g$ - $\gamma$-open sets of $(X, \tau)$ by using the operation $\gamma$ on $\tau_{g}$. Also, some notions of $g$ - $\gamma$-open sets with their relationships are studied. In Section 
4, we introduce the concept of $g \gamma$-generalized closed sets and then investigate some of its properties. In Section 5 , $g$ - $\gamma$ - $T_{i}$ spaces where $i \in\left\{0, \frac{1}{2}, 1,2\right\}$ by utilizing the operation $\gamma$ on $\tau_{g}$ are introduced and investigated. In the last two sections, some basic properties of $g$ - $(\gamma, \beta)$-continuous functions with $g$ - $\beta$-closed graphs have been obtained.

\section{Preliminaries}

Throughout this paper, the space $(X, \tau)$ (or simply $X$ ) always mean topological space on which no separation axioms are assumed unless explicitly stated. For a subset $A$ of a space $X$, the closure of $A$ is denoted by $C l(A)$. A subset $A$ of a topological space $(X, \tau)$ is said to be generalized closed (briefly $g$-closed) [5] if $C l(A) \subseteq U$ whenever $A \subseteq U$ and $U$ is an open set in $X$. The complement of a $g$-closed set of $X$ is $g$-open. The family of all $g$-open subsets of a space $(X, \tau)$ is denoted by $\tau_{g}$. In general, every closed set of a space $X$ is $g$-closed. A space $(X, \tau)$ is $T_{\frac{1}{2}}$ [5] if every $g$-closed subset of $X$ is closed.

Definition 1.[8] An operation $\gamma$ on the topology $\tau$ on $X$ is a mapping $\gamma: \tau \rightarrow P(X)$ such that $U \subseteq \gamma(U)$ for each $U \in \tau$, where $P(X)$ is the power set of $X$ and $\gamma(U)$ denotes the value of $\gamma$ at $U$. A nonempty subset $A$ of a topological space $(X, \tau)$ with an operation $\gamma$ on $\tau$ is said to be $\gamma$-open if for each $x \in A$, there exists an open set $U$ containing $x$ such that $\gamma(U) \subseteq A$. The complement of a $\gamma$-open subset of a space $X$ as $\gamma$-closed. The family of all $\gamma$-open subsets of a space $(X, \tau)$ is denoted by $\tau_{\gamma}$.

Definition 2. [4] A point $x \in X$ is in the $\gamma$-closure of a set $A \subseteq X$ if $\gamma(U) \cap A \neq \phi$ for each open set $U$ containing $x$. The set of all $\gamma$-closure points of $A$ is called $\gamma$-closure of $A$ and is denoted by $C l_{\gamma}(A)$.

Definition 3. [8] A subset $A$ of $(X, \tau)$ with an operation $\gamma$ on $\tau$ is said to be $\gamma$-g-closed if $C l_{\gamma}(A) \subseteq U$ whenever $A \subseteq U$ and $U$ is $\gamma$-open in $(X, \tau)$.

Definition 4. [8] A topological space $(X, \tau)$ with an operation $\gamma$ on $\tau$ is said to be

1. $\gamma-T_{0}$ if for any two distinct points $x, y$ in $X$, there exists an open set $U$ such that $x \in U$ and $y \notin \gamma(U)$ or $y \in U$ and $x \notin \gamma(U)$.

2. $\gamma-T_{1}$ if for any two distinct points $x, y$ in $X$, there exist two open sets $U$ and $V$ containing $x$ and $y$ respectively such that $y \notin \gamma(U)$ and $x \notin \gamma(V)$.

3. $\gamma-T_{2}$ if for any two distinct points $x, y$ in $X$, there exist two open sets $U$ and $V$ containing $x$ and $y$ respectively such that $\gamma(U) \cap \gamma(V)=\phi$.

4. $\gamma$-T $T_{\frac{1}{2}}$ if every $\gamma$-g-closed set in $X$ is $\gamma$-closed.

Theorem 1. [5] If a topological space $(X, \tau)$ is $T_{\frac{1}{2}}$, then $\tau_{g}=\tau$.

\section{$3 g-\gamma$-Open Sets}

Definition 5. An operation $\gamma$ on $\tau_{g}$ is a mapping $\gamma: \tau_{g} \rightarrow P(X)$ such that $U \subseteq \gamma(U)$ for every $U \in \tau_{g}$, where $P(X)$ is the power set of $X$ and $\gamma(U)$ is the value of $\gamma$ at $U$.

From this definition, we can easy to find $\gamma(X)=X$ for any operation $\gamma: \tau_{g} \rightarrow P(X)$.

Definition 6. Let $(X, \tau)$ be a topological space and $\gamma: \tau_{g} \rightarrow P(X)$ be an operation on $\tau_{g}$. A nonempty set A of $X$ is said to be $g$ - $\gamma$-open if for each $x \in A$, there exists a g-open set $U$ such that $x \in U$ and $\gamma(U) \subseteq A$. The complement of a g- $\gamma$-open set of $X$ is $g$ - $\gamma$-closed. Assume that the empty set $\phi$ is also $g$ - $\gamma$-open set for any operation $\gamma: \tau_{g} \rightarrow P(X)$. The family of all $g$ - $\gamma$-open subsets of a space $(X, \tau)$ is denoted by $\tau_{g \gamma}$. 
Theorem 2. The union of any collection of $g$ - $\gamma$-open sets in a topological space $X$ is a $g$ - $\gamma$-open.

Proof. Let $x \in \bigcup_{\lambda \in \Lambda}\left\{A_{\lambda}\right\}$, where $\left\{A_{\lambda}\right\}_{\lambda \in \Lambda}$ be a class of $g$ - $\gamma$-open sets in $X$. Then $x \in A_{\lambda}$ for some $\lambda \in \Lambda$. Since $A_{\lambda}$ is $g$ - $\gamma$-open set in $X$, then there exists a $g$-open set $V$ such that $x \in V \subseteq \gamma(V) \subseteq A_{\lambda} \subseteq \bigcup_{\lambda \in \Lambda}\left\{A_{\lambda}\right\}$. Therefore, $\bigcup_{\lambda \in \Lambda}\left\{A_{\lambda}\right\}$ is $g-\gamma$-open set in $X$.

Example 1. The intersection of any two $g$ - $\gamma$-open sets in $(X, \tau)$ is generally not a $g$ - $\gamma$-open sets. To see this, let $X=\{a, b, c\}$ and $\tau=P(X)=\tau_{g}$. Let $\gamma: \tau_{g} \rightarrow P(X)$ be an operation on $\tau_{g}$ defined as follows:

For every $A \in \tau_{g}$

$$
\gamma(A)= \begin{cases}A & \text { if } A \neq\{c\} \\ \{b, c\} & \text { if } A=\{c\}\end{cases}
$$

Thus, $\tau_{g \gamma}=P(X) \backslash\{c\}$. Then $\{a, c\} \in \tau_{g \gamma}$ and $\{b, c\} \in \tau_{g \gamma}$, but $\{a, c\} \cap\{b, c\}=\{c\} \notin \tau_{g \gamma}$.

Remark. Since the union of two $g$-open sets is generally not a $g$-open set. So the concept of $g$-open set and $g$ - $\gamma$-open set are independent (That is, $\tau_{g} \neq \tau_{g \gamma}$ ). It is shown by the following two examples.

Example 2. In 1, the set $\{c\}$ is $g$-open, but it is not $g$ - $\gamma$-open.

Example 3. Let $X=\{a, b, c\}$ and $\tau=\{\phi, X,\{a\}\}$. Then $\tau_{g}=P(X) \backslash\{b, c\}$. Define an operation $\gamma: \tau_{g} \rightarrow P(X)$ by $\gamma(A)=A$ for all $A \in \tau_{g}$. Here, $\tau_{g \gamma}=P(X)$. Then the set $\{b, c\}$ is $g$ - $\gamma$-open, but $\{b, c\}$ is not a $g$-open set.

Definition 7. A topological space $(X, \tau)$ with an operation $\gamma$ on $\tau_{g}$ is said to be $g-\gamma$-regular iffor each $x \in X$ and for each g-open set $U$ containing $x$, there exists a g-open set $W$ such that $x \in W$ and $\gamma(W) \subseteq U$.

Theorem 3. Let $(X, \tau)$ be a topological space and $\gamma: \tau_{g} \rightarrow P(X)$ be an operation on $\tau_{g}$. Then the following conditions are equivalent:

1. $\tau_{g} \subseteq \tau_{g \gamma}$.

2. $(X, \tau)$ is a $g$ - $\gamma$-regular space.

3.For every $x \in X$ and for every g-open set $U$ of $(X, \tau)$ containing $x$, there exists a g- $\gamma$-open set $W$ of $(X, \tau)$ containing $x$ such that $W \subseteq U$.

Proof.1. $\Rightarrow(2)$ Let $x \in X$ and $U$ be a $g$-open set in $X$ such that $x \in U$. It follows from assumption that $U$ is a $g$ - $\gamma$-open set. This implies that there exists a $g$-open set $W$ such that $x \in W$ and $\gamma(W) \subseteq U$. Therefore, the space $(X, \tau)$ is $g$ - $\gamma$-regular.

2. $\Rightarrow$ (3) Let $x \in X$ and $U$ be a $g$-open set in $(X, \tau)$ containing $x$. Then by (2), there is a $g$-open set $W$ such that $x \in W \subseteq \gamma(W) \subseteq U$. Again, by using (2) for the set $W$, it is shown that $W$ is $g$ - $\gamma$-open. Hence $W$ is a $g$ - $\gamma$-open set containing $x$ such that $W \subseteq U$.

3. $\Rightarrow$ (1) By applying the part (3) and 2, it follows that every $g$-open set of $X$ is $g$ - $\gamma$-open in $X$. That is, $\tau_{g} \subseteq \tau_{g \gamma}$.

Remark. Since every open set is $g$-open. Then by 6 and 1 , every $\gamma$-open set is $g$ - $\gamma$-open (this means that $\tau_{\gamma} \subseteq \tau_{g \gamma}$ ), but the converse is not true in general. For instance, in 3 , we have $\tau_{\gamma}=\tau$. Therefore, the set $\{b\} \in \tau_{g \gamma}$, but the set $\{b\} \notin \tau_{\gamma}$.

Lemma 1. If the space $(X, \tau)$ is $T_{\frac{1}{2}}$, then the concept of $g$ - $\gamma$-open set and $\gamma$-open set coincide (That is $\tau_{g \gamma}=\tau_{\gamma}$ ).

Proof. Follows from their definitions and 1.

Definition 8. Let $(X, \tau)$ be any topological space. An operation $\gamma$ on $\tau_{g}$ is said to be 
1.g-open if for each $x \in X$ and for every g-open set $U$ containing $x$, there exists a $g$ - $\gamma$-open set $W$ containing $x$ such that $W \subseteq \gamma(U)$.

2.g-regular iffor each $x \in X$ and for every pair of g-open sets $U_{1}$ and $U_{2}$ such that both containing $x$, there exists a g-open set $W$ containing $x$ such that $\gamma(W) \subseteq \gamma\left(U_{1}\right) \cap \gamma\left(U_{2}\right)$.

Proposition 1. Let a mapping $\gamma$ be g-regular operation on $\tau_{g}$. If the subsets $A$ and $B$ are $g$ - $\gamma$-open in a topological space $(X, \tau)$, then $A \cap B$ is also $g$ - $\gamma$-open set in $(X, \tau)$.

Proof. Suppose $x \in A \cap B$ for any $g$ - $\gamma$-open subsets $A$ and $B$ in $(X, \tau)$ both containing $x$. Then there exist $g$-open sets $U_{1}$ and $U_{2}$ such that $x \in U_{1} \subseteq A$ and $x \in U_{2} \subseteq B$. Since $\gamma$ is a $g$-regular operation on $\tau_{g}$, then there exists a $g$-open set $W$ containing $x$ such that $\gamma(W) \subseteq \gamma\left(U_{1}\right) \cap \gamma\left(U_{2}\right) \subseteq A \cap B$. Therefore, $A \cap B$ is $g$ - $\gamma$-open set in $(X, \tau)$.

Remark. By applying 1 , it is easy to show that $\tau_{g \gamma}$ forms a topology on $X$ for any $g$-regular operation $\gamma$ on $\tau_{g}$.

Definition 9. The point $x \in X$ is in the g-closure $\gamma$ of a set $A$ if $\gamma(U) \cap A \neq \phi$ for each $g$-open set $U$ containing $x$. The set of all $g$-closure $\gamma$ points of $A$ is called $g$-closure $\gamma$ of $A$ and is denoted by $g C l_{\gamma}(A)$.

Definition 10. Let $A$ be any subset of a topological space $(X, \tau)$ and $\gamma$ be an operation on $\tau_{g}$. The $g$ - $\gamma$-closure of $A$ is defined as the intersection of all $g$ - $\gamma$-closed sets of $X$ containing $A$ and it is denoted by $g_{\gamma} C l(A)$. That is, $g_{\gamma} C l(A)=\bigcap\left\{F: A \subseteq F, X \backslash F \in \tau_{g \gamma}\right\}$.

Theorem 4. Let $A$ be any subset of a topological space $(X, \tau)$ and $\gamma$ be an operation on $\tau_{g}$. Then $x \in g_{\gamma} C l(A)$ if and only if $A \cap U \neq \phi$ for every $g$ - $\gamma$-open set $U$ of $X$ containing $x$.

Proof. Let $x \in g_{\gamma} C l(A)$ and let $A \cap U=\phi$ for some $g$ - $\gamma$-open set $U$ of $X$ containing $x$. Then $A \subseteq X \backslash U$ and $X \backslash U$ is $g$ - $\gamma$-closed set in $X$. So $g_{\gamma} C l(A) \subseteq X \backslash U$. Thus, $x \in X \backslash U$. This is a contradiction. Hence $A \cap U \neq \phi$ for every $g$ - $\gamma$-open set $U$ of $X$ containing $x$.

Conversely, suppose that $x \notin g_{\gamma} C l(A)$. So there exists a $g$ - $\gamma$-closed set $F$ such that $A \subseteq F$ and $x \notin F$. Then $X \backslash F$ is a $g$ - $\gamma$-open set such that $x \in X \backslash F$ and $A \cap(X \backslash F)=\phi$. Contradiction of hypothesis. Therefore, $x \in g_{\gamma} C l(A)$.

Lemma 2. The following statements are true for any subsets $A$ and $B$ of a topological space $(X, \tau)$ with an operation $\gamma$ on $\tau_{g}$.

1. $g_{\gamma} \mathrm{Cl}(A)$ is $g$ - $\gamma$-closed set in $\mathrm{X}$ and $g \mathrm{Cl}_{\gamma}(A)$ is $\mathrm{g}$-closed set in $\mathrm{X}$.

2.A $\subseteq g C l_{\gamma}(A) \subseteq g_{\gamma} C l(A)$.

$3 . g_{\gamma} C l(\phi)=g C l_{\gamma}(\phi)=\phi$ and $g_{\gamma} C l(X)=g C l_{\gamma}(X)=X$.

(a)A is $g$ - $\gamma$-closed if and only if $g_{\gamma} C l(A)=A$ and,

(b)A is $g$ - $\gamma$-closed if and only if $g C l_{\gamma}(A)=A$.

4.If $A \subseteq B$, then $g_{\gamma} C l(A) \subseteq g_{\gamma} C l(B)$ and $g C l_{\gamma}(A) \subseteq g C l_{\gamma}(B)$.

(a) $g_{\gamma} C l(A \cap B) \subseteq g_{\gamma} C l(A) \cap g_{\gamma} C l(B)$ and,

(b) $g C l_{\gamma}(A \cap B) \subseteq g C l_{\gamma}(A) \cap g C l_{\gamma}(B)$.

(c) $g_{\gamma} C l(A) \cup g_{\gamma} C l(B) \subseteq g_{\gamma} C l(A \cup B)$ and,

(d) $g C l_{\gamma}(A) \cup g C l_{\gamma}(B) \subseteq g C l_{\gamma}(A \cup B)$.

$5 . g_{\gamma} C l\left(g_{\gamma} C l(A)\right)=g_{\gamma} C l(A)$.

Proof. Straightforward.

Theorem 5. For any subsets $A, B$ of a topological space $(X, \tau)$. If $\gamma$ is a g-regular operation on $\tau_{g}$, then

$1 . g_{\gamma} C l(A) \cup g_{\gamma} C l(B)=g_{\gamma} C l(A \cup B)$. 
2. $g C l_{\gamma}(A) \cup g C l_{\gamma}(B)=g C l_{\gamma}(A \cup B)$.

Proof.1.It is enough to proof that $g_{\gamma} C l(A \cup B) \subseteq g_{\gamma} C l(A) \cup g_{\gamma} C l(B)$ since the other part follows directly from 2 (7). Let $x \notin g_{\gamma} C l(A) \cup g_{\gamma} C l(B)$. Then there exist two $g$ - $\gamma$-open sets $U$ and $V$ containing $x$ such that $A \cap U=\phi$ and $B \cap V=\phi$. Since $\gamma$ is a $g$-regular operation on $\tau_{g}$, then by $1, U \cap V$ is $g$ - $\gamma$-open in $X$ such that

$$
(U \cap V) \cap(A \cup B)=\phi .
$$

Therefore, we have $x \notin g_{\gamma} C l(A \cup B)$ and hence

$$
g_{\gamma} C l(A \cup B) \subseteq g_{\gamma} C l(A) \cup g_{\gamma} C l(B) .
$$

2.Let $x \notin g C l_{\gamma}(A) \cup g C l_{\gamma}(B)$. Then there exist $g$-open sets $U_{1}$ and $U_{2}$ such that $x \in U_{1}, x \in U_{2}, A \cap \gamma\left(U_{1}\right)=\phi$ and $A$ $\cap \gamma\left(U_{2}\right)=\phi$. Since $\gamma$ is a $g$-regular operation on $\tau_{g}$, then there exists a $g$-open set $W$ containing $x$ such that $\gamma(W) \subseteq$ $\gamma\left(U_{1}\right) \cap \gamma\left(U_{2}\right)$. Thus, we have

$$
(A \cup B) \cap \gamma(W) \subseteq(A \cup B) \cap\left(\gamma\left(U_{1}\right) \cap \gamma\left(U_{2}\right)\right) .
$$

This implies that $(A \cup B) \cap \gamma(W)=\phi$ since $(A \cup B) \cap\left(\gamma\left(U_{1}\right) \cap \gamma\left(U_{2}\right)\right)=\phi$. This means that $x \notin g C l_{\gamma}(A \cup B)$ and hence $\operatorname{gCl}_{\gamma}(A \cup B) \subseteq g \operatorname{gl}_{\gamma}(A) \cup g C l_{\gamma}(B)$. Using 2 (7), we have the equality.

Theorem 6. Let $A$ be any subset of a topological space $(X, \tau)$. If $\gamma$ is a g-open operation on $\tau_{g}$, then $g C l_{\gamma}(A)=g_{\gamma} C l(A)$, $g C l_{\gamma}\left(g C l_{\gamma}(A)\right)=g C l_{\gamma}(A)$ and $g C l_{\gamma}(A)$ is $g$ - $\gamma$-closed set in $X$.

Proof. First we need to show that $g_{\gamma} C l(A) \subseteq g C l_{\gamma}(A)$ since by 2 (2), we have $g C l_{\gamma}(A) \subseteq g_{\gamma} C l(A)$. Now let $x \notin g C l_{\gamma}(A)$, then there exists a $g$-open set $U$ containing $x$ such that $A \cap \gamma(U)=\phi$. Since $\gamma$ is a $g$-open on $\tau_{g}$, then there exists a $g-\gamma$ open set $W$ containing $x$ such that $W \subseteq \gamma(U)$. So $A \cap W=\phi$ and hence by $4, x \notin g_{\gamma} C l(A)$. Therefore, $g_{\gamma} C l(A) \subseteq g C l_{\gamma}(A)$. Hence $g C l_{\gamma}(A)=g_{\gamma} C l(A)$. Moreover, using the above result and by $2(8)$, we get $g C l_{\gamma}\left(g C l_{\gamma}(A)\right)=g C l_{\gamma}(A)$ and by $2(4 \mathrm{~b})$, we obtain $\mathrm{gCl}_{\gamma}(A)$ is $g$ - $\gamma$-closed set in $X$.

Theorem 7. Let $A$ be any subset of a topological space $(X, \tau)$ and $\gamma$ be an operation on $\tau_{g}$. Then the following statements are equivalent.

1. A is g- $\gamma$-open set.

2. $g C l_{\gamma}(X \backslash A)=X \backslash A$.

3. $g_{\gamma} C l(X \backslash A)=X \backslash A$.

4. $X \backslash A$ is $g$ - $\gamma$-closed set.

Proof. Clear.

Definition 11. A subset $N$ of a topological space $(X, \tau)$ is called a $g-\gamma$-neighbourhood of a point $x \in X$, if there exists a $g$ - $\gamma$-open set $U$ in $X$ such that $x \in U \subseteq N$.

Lemma 3. Let $U \subseteq(X, \tau)$ be a $g$ - $\gamma$-open if and only if it is a $g$ - $\gamma$-neighbourhood of each of its points.

Proof. Let $U$ be any $g-\gamma$-open set in $(X, \tau)$. Then by 11 , it is clear that $U$ is a $g$ - $\gamma$-neighbourhood of each of its points, since for every $x \in U, x \in U \subseteq U$ and $U \in \tau_{g \gamma}$.

Conversely, suppose $U$ is a $g$ - $\gamma$-neighbourhood of each of its points. Then for each $x \in U$, there exists a $g$ - $\gamma$-open set $V_{x}$ containing $x$ such that $V_{x} \subseteq U$. Then $U=\bigcup_{x \in U} V_{x}$. Since each $V_{x}$ is $g$ - $\gamma$-open. It follows from 2 that $U$ is $g-\gamma$-open set in $X$. 
Definition 12. Let $A$ be any subset of a topological space $(X, \tau)$ and $\gamma$ be an operation on $\tau_{g}$. The g- $\gamma$-interior of $A$ is defined as the union of all $g-\gamma$-open sets of $X$ contained in A and it is denoted by $g_{\gamma} I n t(A)$. That is,

$$
g_{\gamma} \operatorname{Int}(A)=\bigcup\left\{U: U \in \tau_{g \gamma} \text { and } U \subseteq A\right\}
$$

Some important properties of $g$ - $\gamma$-interior operator will be given in 4 .

Lemma 4. Let $A$ and $B$ be subset of a topological space $(X, \tau)$ and $\gamma$ be an operation on $\tau_{g}$. Then the following conditions hold.

1. $g_{\gamma} \operatorname{Int}(A)$ is $g$ - $\gamma$-open set in $X$ and $g_{\gamma} \operatorname{Int}(A) \subseteq A$.

2. $g_{\gamma} \operatorname{Int}(\phi)=\phi$ and $g_{\gamma} \operatorname{Int}(X)=X$.

3. $A$ is $g$ - $\gamma$-open if and only if $g_{\gamma} \operatorname{Int}(A)=A$.

4. If $A \subseteq B$, then $g_{\gamma} \operatorname{Int}(A) \subseteq g_{\gamma} \operatorname{Int}(B)$.

5. $g_{\gamma} \operatorname{Int}(A \cap B) \subseteq g_{\gamma} \operatorname{Int}(A) \cap g_{\gamma} \operatorname{Int}(B)$.

6. $g_{\gamma} \operatorname{Int}(A) \cup g_{\gamma} \operatorname{Int}(B) \subseteq g_{\gamma} \operatorname{Int}(A \cup B)$.

7. $g_{\gamma} \operatorname{Int}\left(g_{\gamma} \operatorname{Int}(A)\right)=g_{\gamma} \operatorname{Int}(A)$.

8. $g_{\gamma} \operatorname{Int}(X \backslash A)=X \backslash g_{\gamma} C l(A)$.

Proof. Straightforward.

Theorem 8. If $\gamma$ is a g-regular operation on $\tau_{g}$, then for any subsets $A, B$ of a space $X$, we have

$$
g_{\gamma} \operatorname{Int}(A) \cap g_{\gamma} \operatorname{Int}(B)=g_{\gamma} \operatorname{Int}(A \cap B) .
$$

Proof. Follows directly from 5 (1) and using 4 (8).

Lemma 5. Let $(X, \tau)$ be a topological space and $\gamma$ be a g-regular operation on $\tau_{g}$. Then $g_{\gamma} C l(A) \cap U \subseteq g_{\gamma} C l(A \cap U)$ holds for every $g$ - $\gamma$-open set $U$ and every subset $A$ of $X$.

Proof. Suppose that $x \in g_{\gamma} C l(A) \cap U$ for every $g$ - $\gamma$-open set $U$, then $x \in g_{\gamma} C l(A)$ and $x \in U$. Let $V$ be any $g$ - $\gamma$-open set of $X$ containing $x$. Since $\gamma$ is $g$-regular on $\tau_{g}$. So by $1, U \cap V$ is $g$ - $\gamma$-open set containing $x$. Since $x \in g_{\gamma} C l(A)$, then by 4 , we have $A \cap(U \cap V) \neq \phi$. This means that $(A \cap U) \cap V \neq \phi$. Therefore, again by 4, we obtain that $x \in g_{\gamma} C l(A \cap U)$. Thus, $g_{\gamma} C l(A) \cap U \subseteq g_{\gamma} C l(A \cap U)$.

The proof of the following lemma is similar to 5 and using 4 (8).

Lemma 6. Let $(X, \tau)$ be a topological space and $\gamma$ be a g-regular operation on $\tau_{g}$. Then $g_{\gamma} \operatorname{Int}(A \cup F) \subseteq g_{\gamma} \operatorname{Int}(A) \cup F$ holds for every $g$ - $\gamma$-closed set $F$ and every subset $A$ of $X$.

\section{$4 g \gamma g$-closed sets}

Definition 13. A subset A of a topological space $(X, \tau)$ with an operation $\gamma$ on $\tau_{g}$ is said to be g $\gamma$-generalized closed (briefly grg-closed) if $g C l_{\gamma}(A) \subseteq U$ whenever $A \subseteq U$ and $U$ is a $g$ - $\gamma$-open set in $X$.

Lemma 7. Let $(X, \tau)$ be a topological space and $\gamma$ be an operation on $\tau_{g}$. A set $A$ in $(X, \tau)$ is grg-closed if and only if $A \cap g_{\gamma} C l(\{x\}) \neq \phi$ for every $x \in g C l_{\gamma}(A)$.

Proof. Suppose $A$ is $g \gamma g$-closed set in $X$ and suppose (if possible) that there exists an element $x \in g C l_{\gamma}(A)$ such that $A \cap g_{\gamma} C l(\{x\})=\phi$. This follows that $A \subseteq X \backslash g_{\gamma} C l(\{x\})$. Since $g_{\gamma} C l(\{x\})$ is $g$ - $\gamma$-closed implies $X \backslash g_{\gamma} C l(\{x\})$ is $g$ - $\gamma$-open 
and $A$ is $g \gamma g$-closed set in $X$. Then, we have that $g C l_{\gamma}(A) \subseteq X \backslash g_{\gamma} C l(\{x\})$. This means that $x \notin g C l_{\gamma}(A)$. This is a contradiction. Hence $A \cap g_{\gamma} C l(\{x\}) \neq \phi$.

Conversely, let $U \in \tau_{g \gamma}$ such that $A \subseteq U$. To show that $g C l_{\gamma}(A) \subseteq U$. Let $x \in g C l_{\gamma}(A)$. Then by hypothesis, $A \cap g_{\gamma} C l(\{x\}) \neq \phi$. So there exists an element $y \in A \cap g_{\gamma} C l(\{x\})$. Thus $y \in A \subseteq U$ and $y \in g_{\gamma} C l(\{x\})$. By $4,\{x\} \cap U \neq \phi$. Hence $x \in U$ and so $g C l_{\gamma}(A) \subseteq U$. Therefore, $A$ is $g \gamma g$-closed set in $(X, \tau)$.

Theorem 9. Let $A$ be a subset of topological space $(X, \tau)$ and $\gamma$ be an operation on $\tau_{g}$. If $A$ is g g $g$-closed, then $g C l_{\gamma}(A) \backslash A$ does not contain any non-empty $g$ - $\gamma$-closed set.

Proof. Let $F$ be a non-empty $g$ - $\gamma$-closed set in $X$ such that $F \subseteq g C l_{\gamma}(A) \backslash A$. Then $F \subseteq X \backslash A$ implies $A \subseteq X \backslash F$. Since $X \backslash F$ is $g$ - $\gamma$-open set and $A$ is $g \gamma g$-closed set, then $g C l_{\gamma}(A) \subseteq X \backslash F$. That is $F \subseteq X \backslash g C l_{\gamma}(A)$. Hence $F \subseteq X \backslash g C l_{\gamma}(A) \cap g C l_{\gamma}(A) \backslash A \subseteq$ $X \backslash g C l_{\gamma}(A) \cap g C l_{\gamma}(A)=\phi$. This shows that $F=\phi$. This is contradiction. Therefore, $F \nsubseteq g C l_{\gamma}(A) \backslash A$.

Theorem 10. If $\gamma: \tau_{g} \rightarrow P(X)$ is a g-open operation, then the converse of the 9 is true.

Proof. Let $U$ be a $g$ - $\gamma$-open set in $(X, \tau)$ such that $A \subseteq U$. Since $\gamma: \tau_{g} \rightarrow P(X)$ is a $g$-open operation, then by $6, g C l_{\gamma}(A)$ is $g$ $\gamma$-closed set in $X$. Thus, using 2 , we have $g C l_{\gamma}(A) \cap X \backslash U$ is a $g$ - $\gamma$-closed set in $(X, \tau)$. Since $X \backslash U \subseteq X \backslash A, g C l_{\gamma}(A) \cap X \backslash U \subseteq$ $g C l_{\gamma}(A) \backslash A$. Using the assumption of the converse of the $9, g C l_{\gamma}(A) \subseteq U$. Therefore, $A$ is $g \gamma g$-closed set in $(X, \tau)$.

Corollary 1. Let $A$ be a g $\gamma g$-closed subset of topological space $(X, \tau)$ and let $\gamma$ be an operation on $\tau_{g}$. Then $A$ is $g$ - $\gamma$-closed if and only if $g \mathrm{Cl}_{\gamma}(A) \backslash A$ is $g$ - $\gamma$-closed set.

Proof. Let $A$ be a $g$ - $\gamma$-closed set in $(X, \tau)$. Then by 2 (4b), $g C l_{\gamma}(A)=A$ and hence $g C l_{\gamma}(A) \backslash A=\phi$ which is $g$ - $\gamma$-closed set.

Conversely, suppose $g C l_{\gamma}(A) \backslash A$ is $g$ - $\gamma$-closed and $A$ is $g \gamma g$-closed. Then by $9, g C l_{\gamma}(A) \backslash A$ does not contain any non-empty $g$ - $\gamma$-closed set and since $g C l_{\gamma}(A) \backslash A$ is $g$ - $\gamma$-closed subset of itself, then $g C l_{\gamma}(A) \backslash A=\phi$ implies $g C l_{\gamma}(A) \cap X \backslash A=\phi$. Hence $g C l_{\gamma}(A)=A$. This follows from 2 (4b) that $A$ is $g$ - $\gamma$-closed set in $(X, \tau)$.

Theorem 11. Let $(X, \tau)$ be a topological space and $\gamma$ be an operation on $\tau_{g}$. If a subset $A$ of $X$ is g $\gamma g$-closed and $g$ - $\gamma$-open, then $A$ is $g$ - $\gamma$-closed.

Proof. Since $A$ is $g \gamma g$-closed and $g$ - $\gamma$-open set in $X$, then $g C l_{\gamma}(A) \subseteq A$ and hence by 2 (4b), $A$ is $g$ - $\gamma$-closed.

Theorem 12. In any topological space $(X, \tau)$ with an operation $\gamma$ on $\tau_{g}$. For an element $x \in X$, the set $X \backslash\{x\}$ is g g g-closed or g- $\gamma$-open.

Proof. Suppose that $X \backslash\{x\}$ is not $g$ - $\gamma$-open. Then $X$ is the only $g$ - $\gamma$-open set containing $X \backslash\{x\}$. This implies that $g C l_{\gamma}(X \backslash\{x\}) \subseteq X$. Thus $X \backslash\{x\}$ is a $g \gamma g$-closed set in $X$.

Corollary 2. In any topological space $(X, \tau)$ with an operation $\gamma$ on $\tau_{g}$. For an element $x \in X$, either the set $\{x\}$ is $g$ - $\gamma$-closed or the set $X \backslash\{x\}$ is grg-closed.

Proof. Suppose $\{x\}$ is not $g$ - $\gamma$-closed, then $X \backslash\{x\}$ is not $g$ - $\gamma$-open. Hence by $12, X \backslash\{x\}$ is $g \gamma g$-closed set in $X$.

Definition 14. Let $A$ be any subset of a topological space $(X, \tau)$ and $\gamma$ be an operation on $\tau_{g}$. Then the $\tau_{g \gamma}$-kernel of $A$ is denoted by $\tau_{g \gamma-k e r}(A)$ and is defined as follows.

$$
\tau_{g \gamma}-\operatorname{ker}(A)=\cap\left\{U: A \subseteq U \text { and } U \in \tau_{g \gamma}\right\}
$$

In other words, $\tau_{g \gamma-k e r}(A)$ is the intersection of all g- $\gamma$-open sets of $(X, \tau)$ containing $A$. 
Theorem 13. Let $A \subseteq(X, \tau)$ and $\gamma$ be an operation on $\tau_{g}$. Then $A$ is $g \gamma g$-closed if and only if $g C l_{\gamma}(A) \subseteq \tau_{g \gamma}-k e r(A)$.

Proof. Suppose that $A$ is $g \gamma g$-closed. Then $g C l_{\gamma}(A) \subseteq U$, whenever $A \subseteq U$ and $U$ is $g$ - $\gamma$-open. Let $x \in g C l_{\gamma}(A)$. Then by 7, $A \cap g_{\gamma} C l(\{x\}) \neq \phi$. So there exists a point $z$ in $X$ such that $z \in A \cap g_{\gamma} C l(\{x\})$ implies that $z \in A \subseteq U$ and $z \in g_{\gamma} C l(\{x\})$. By $4,\{x\} \cap U \neq \phi$. Hence we show that $x \in \tau_{g \gamma}$ - $\operatorname{ker}(A)$. Therefore, $g C l_{\gamma}(A) \subseteq \tau_{g \gamma}-\operatorname{ker}(A)$.

Conversely, let $g C l_{\gamma}(A) \subseteq \tau_{g \gamma}$-ker(A). Let $U$ be any $g$ - $\gamma$-open set containing $A$. Let $x$ be a point in $X$ such that $x \in g C l_{\gamma}(A)$. Then $x \in \tau_{g \gamma}-\operatorname{ker}(A)$. Namely, we have $x \in U$, because $A \subseteq U$ and $\left.U \in \tau_{g \gamma}\right\}$. That is $g C l_{\gamma}(A) \subseteq \tau_{g \gamma}-\operatorname{ker}(A) \subseteq U$. Therefore, $A$ is $g \gamma g$-closed set in $X$.

\section{$5 g-\gamma-T_{i}$ Spaces for $i \in\left\{0, \frac{1}{2}, 1,2\right\}$}

In this section, we introduce some types of $g$ - $\gamma$ - separation axioms called $g-\gamma$ - $T_{i}$ for $i \in\left\{0, \frac{1}{2}, 1,2\right\}$. Some basic properties of these spaces are investigated.

Definition 15. A topological space $(X, \tau)$ with an operation $\gamma$ on $\tau_{g}$ is said to be $g-\gamma-T_{0}$ if for any two distinct points $x, y$ in $X$, there exists a g-open set $U$ such that $x \in U$ and $y \notin \gamma(U)$ or $y \in U$ and $x \notin \gamma(U)$.

Definition 16. A topological space $(X, \tau)$ with an operation $\gamma$ on $\tau_{g}$ is said to be $g-\gamma-T_{1}$ if for any two distinct points $x, y$ in $X$, there exist two g-open sets $U$ and $V$ containing $x$ and $y$ respectively such that $y \notin \gamma(U)$ and $x \notin \gamma(V)$.

Definition 17. A topological space $(X, \tau)$ with an operation $\gamma$ on $\tau_{g}$ is said to be $g-\gamma-T_{2}$ if for any two distinct points $x, y$ in $X$, there exist two g-open sets $U$ and $V$ containing $x$ and y respectively such that $\gamma(U) \cap \gamma(V)=\phi$.

Definition 18. A topological space $(X, \tau)$ with an operation $\gamma$ on $\tau_{g}$ is said to be $g-\gamma$ - $T_{\frac{1}{2}}$ if every g $\gamma g$-closed set in $X$ is g- $\gamma$-closed set.

Theorem 14. For any topological space $(X, \tau)$ with an operation $\gamma$ on $\tau_{g}$. Then $(X, \tau)$ is $g-\gamma-T_{\frac{1}{2}}$ if and only if for each element $x \in X$, the set $\{x\}$ is $g$ - $\gamma$-closed or $g$ - $\gamma$-open.

Proof. Let $X$ be a $g-\gamma$ - $T_{\frac{1}{2}}$ space and let $\{x\}$ is not $g$ - $\gamma$-closed set in $(X, \tau)$. By $2, X \backslash\{x\}$ is $g \gamma g$-closed. Since $(X, \tau)$ is $g-\gamma-T_{\frac{1}{2}}$, then $X \backslash\{x\}$ is $g$ - $\gamma$-closed set which means that $\{x\}$ is $g$ - $\gamma$-open set in $X$.

Conversely, let $F$ be any $g \gamma g$-closed set in the space $(X, \tau)$. We have to show that $F$ is $g$ - $\gamma$-closed (that is $g C l_{\gamma}(F)=F$ (by 2 (4b))). It is sufficient to show that $g C l_{\gamma}(F) \subseteq F$. Let $x \in g C l_{\gamma}(F)$. By hypothesis $\{x\}$ is $g$ - $\gamma$-closed or $g$ - $\gamma$-open for each $x \in X$. So we have two cases.

Case 1. If $\{x\}$ is $g$ - $\gamma$-closed set. Suppose $x \notin F$, then $x \in g C l_{\gamma}(F) \backslash F$ contains a non-empty $g$ - $\gamma$-closed set $\{x\}$. A contradiction since $F$ is $g \gamma g$-closed set and according to the 9. Hence $x \in F$. This follows that $g C l_{\gamma}(F) \subseteq F$ and hence $g C l_{\gamma}(F)=F$. This means from by $2(4 \mathrm{~b})$ that $F$ is $g$ - $\gamma$-closed set in $(X, \tau)$. Thus $(X, \tau)$ is $g-\gamma-T_{\frac{1}{2}}$ space.

Case 1. If $\{x\}$ is $g$ - $\gamma$-open set. Then by $4, F \cap\{x\} \neq \phi$ which implies that $x \in F$. So $g C l_{\gamma}(F) \subseteq F$. Thus by 2 (4b), $F$ is $g$ - $\gamma$-closed. Therefore, $(X, \tau)$ is $g-\gamma-T_{\frac{1}{2}}$ space.

Theorem 15. Let $\gamma$ be a g-open operation on $\tau_{g}$. Then $(X, \tau)$ is a $g-\gamma-T_{0}$ space if and only if $g C l_{\gamma}(\{x\}) \neq g C l_{\gamma}(\{y\})$, for every pair $x$, $y$ of $X$ with $x \neq y$. 
Proof. Necessary Part. Let $x, y$ be any two distinct points of a $g-\gamma-T_{0}$ space $(X, \tau)$. Then by definition, we assume that there exists a $g$ - $\gamma$-open set $U$ such that $x \in U$ and $y \notin \gamma(U)$. Since $\gamma$ is a $g$-open operation on $\tau_{g}$, then there exists a $g$ - $\gamma$-open set $W$ such that $x \in W$ and $W \subseteq \gamma(U)$. Hence $y \in X \backslash \gamma(U) \subseteq X \backslash W$. Since $X \backslash W$ is a $g$ - $\gamma$-closed set in $(X, \tau)$. Then we obtain that $g C l_{\gamma}(\{y\}) \subseteq X \backslash W$ and therefore $g C l_{\gamma}(\{x\}) \neq g C l_{\gamma}(\{y\})$.

Sufficient Part. Suppose for any $x, y \in X$ with $x \neq y$, we have $g C l_{\gamma}(\{x\}) \neq g C l_{\gamma}(\{y\})$. Now, we assume that there exists $z \in X$ such that $z \in g C l_{\gamma}(\{x\})$, but $z \notin g C l_{\gamma}(\{y\})$. If $x \in g C l_{\gamma}(\{y\})$, then $\{x\} \subseteq g C l_{\gamma}(\{y\})$, which implies that $g C l_{\gamma}(\{x\}) \subseteq g C l_{\gamma}(\{y\})$ (by $\left.2(5)\right)$. This implies that $z \in g C l_{\gamma}(\{y\})$. This contradiction shows that $x \notin g C l_{\gamma}(\{y\})$. This means that by 9 , there exists a $g$-open set $U$ such that $x \in U$ and $\gamma(U) \cap\{y\}=\phi$. Thus, we have that $x \in U$ and $y \notin \gamma(U)$. It gives that the space $(X, \tau)$ is $g-\gamma-T_{0}$.

Theorem 16. The space $(X, \tau)$ is $g-\gamma-T_{1}$ if and only if for every point $x \in X,\{x\}$ is a $g$ - $\gamma$-closed set.

Proof. Necessary Part. Let $x$ be a point of a $g-\gamma-T_{1}$ space $(X, \tau)$. Then for any point $y \in X$ such that $x \neq y$, there exists a $g$-open set $V_{y}$ such that $y \in V_{y}$ but $x \notin \gamma\left(V_{y}\right)$. Thus, $y \in \gamma\left(V_{y}\right) \subseteq X \backslash\{x\}$. This implies that $X \backslash\{x\}=\cup\left\{\gamma\left(V_{y}\right): y \in X \backslash\{x\}\right\}$. It is shown that $X \backslash\{x\}$ is $g$ - $\gamma$-open set in $(X, \tau)$. Hence $\{x\}$ is $g$ - $\gamma$-closed set in $(X, \tau)$.

Sufficient Part. Let $x, y \in X$ such that $x \neq y$. By hypothesis, we get $X \backslash\{y\}$ and $X \backslash\{x\}$ are $g$ - $\gamma$-open sets such that $x \in X \backslash\{y\}$ and $y \in X \backslash\{x\}$. Therefore, there exist $g$-open sets $U$ and $V$ such that $x \in U, y \in V, \gamma(U) \subseteq X \backslash\{y\}$ and $\gamma(V) \subseteq X \backslash\{x\}$. So, $y \notin \gamma(U)$ and $x \notin \gamma(V)$. This implies that $(X, \tau)$ is $g-\gamma-T_{1}$.

Theorem 17. For any topological space $(X, \tau)$ and any operation $\gamma$ on $\tau_{g}$, the following properties hold.

1. Every $g-\gamma$ - $T_{2}$ space is $g-\gamma-T_{1}$.

2. Every $g-\gamma-T_{1}$ space is $g-\gamma-T_{1}$.

3. Every $g-\gamma$ - $T_{\frac{1}{2}}$ space is $g-\gamma-T_{0}$.

Proof. The proofs are obvious by their definitions.

Remark. By 17, 3 and [8], we obtain the following diagram of implications. Moreover, the following ?? below show that the reverse implications are not true in general.

$$
\begin{gathered}
g-\gamma-T_{2} \rightarrow g-\gamma-T_{1} \rightarrow g-\gamma-T_{\frac{1}{2}} \rightarrow g-\gamma-T_{0} \\
\gamma-T_{2} \rightarrow \gamma-T_{1} \rightarrow \gamma-T_{\frac{1}{2}} \rightarrow \gamma-T_{0}
\end{gathered}
$$

Example 4. Let $X=\{a, b, c\}$ and $\tau=\{\phi, X\}$. Then $\tau_{g}=P(X)$. Define an operation $\gamma: \tau_{g} \rightarrow P(X)$ by $\gamma(A)=A$ for all $A \in \tau_{g}$. Here, $\tau_{g \gamma}=P(X)$ and $\tau=\tau_{\gamma}$. Then the space $(X, \tau)$ is $g-\gamma-T_{i}\left(i \in\left\{0, \frac{1}{2}, 1,2\right\}\right)$, but not $\gamma-T_{i}\left(i \in\left\{0, \frac{1}{2}, 1,2\right\}\right)$.

Example 5. Let $X=\{a, b, c\}$ and $\tau=\{\phi, X,\{a\},\{a, b\}\}$. Then $\tau_{g}=\{\phi, X,\{a\},\{b\},\{a, b\}\}$. Let $\gamma: \tau_{g} \rightarrow P(X)$ be an operation on $\tau_{g}$ defined as follows. For every set $A \in \tau_{g}$

$$
\gamma(A)= \begin{cases}A & \text { if } b \in A \\ C l(A) & \text { if } b \notin A\end{cases}
$$

Thus, $\tau_{g \gamma}=\{\phi, X,\{b\},\{a, b\}\}$. Then the space $(X, \tau)$ is $g-\gamma-T_{0}$, but it is not $g-\gamma-T_{\frac{1}{2}}$. Since $\{b, c\}$ is $g \gamma g$-closed set in $(X, \tau)$, but $\{b, c\}$ is not $g$ - $\gamma$-closed set in $(X, \tau)$.

Example 6. Let $X=\{a, b, c\}$ and $\tau=\{\phi, X,\{a\},\{b\},\{a, b\},\{a, c\}\}$. Then $\tau_{g}=\tau$. Let $\gamma: \tau_{g} \rightarrow P(X)$ be an operation on $\tau_{g}$ defined as follows: 
For every set $A \in \tau_{g}$

$$
\gamma(A)= \begin{cases}A, & \text { if } a \in A \\ C l(A), & \text { if } a \notin A\end{cases}
$$

Thus, $\tau_{g \gamma}=\tau$. Therefore, the space $(X, \tau)$ is $g-\gamma-T_{\frac{1}{2}}$, but it is not $g-\gamma-T_{1}$.

Example 7. Suppose $X=\{a, b, c\}$ and $\tau$ be the discrete topology on $X$. Define an operation $\gamma$ on $\tau_{g}$ as follows. For every $A \in \tau_{g}$

$$
\gamma(A)=\left\{\begin{array}{l}
A, \text { if } A=\{a, b\} \text { or }\{a, c\} \text { or }\{b, c\} \\
X, \text { otherwise }
\end{array}\right.
$$

Then $(X, \tau)$ is $g-\gamma-T_{1}$ space, but $(X, \tau)$ is not $g-\gamma-T_{2}$.

Lemma 8. Let $(X, \tau)$ be a $T_{\frac{1}{2}}$ space. Then $(X, \tau)$ is $g-\gamma-T_{i}$ if and only if it is $\gamma-T_{i}$, where $i \in\{0,1,2\}$.

\section{$6 g-(\gamma, \beta)$-Continuous Functions}

Throughout Section 6 and Section 7 , let $(X, \tau)$ and $(Y, \sigma)$ be two topological spaces and let $\gamma: \tau_{g} \rightarrow P(X)$ and $\beta: \sigma_{g} \rightarrow$ $P(Y)$ be operations on $\tau_{g}$ and $\sigma_{g}$ respectively. In this section, we introduce a new class of functions called $g$ - $(\gamma, \beta)$ continuous. Some characterizations and properties of this function are investigated.

Definition 19. A function $f:(X, \tau) \rightarrow(Y, \sigma)$ is said to be $g$ - $(\gamma, \beta)$-continuous if for each $x \in X$ and each g-open set $V$ containing $f(x)$, there exists a g-open set $U$ containing $x$ such that $f(\gamma(U)) \subseteq \beta(V)$.

Theorem 18. Let $f:(X, \tau) \rightarrow(Y, \sigma)$ be a $g$ - $(\gamma, \beta)$-continuous function, then,

1. $f\left(g C l_{\gamma}(A)\right) \subseteq g C l_{\beta}(f(A))$, for every $A \subseteq(X, \tau)$.

2. $f^{-1}(F)$ is $g$ - $\gamma$-closed set in $(X, \tau)$, for every $g$ - $\beta$-closed set $F$ of $(Y, \sigma)$.

Proof.1. Let $y \in f\left(g C l_{\gamma}(A)\right)$ and $V$ be any $g$-open set containing $y$. Then by hypothesis, there exists $x \in X$ and $g$-open set $U$ containing $x$ such that $f(x)=y$ and $f(\gamma(U)) \subseteq \beta(V)$. Since $x \in g C l_{\gamma}(A)$, we have $\gamma(U) \cap A \neq \phi$. Hence $\phi \neq$ $f(\gamma(U) \cap A) \subseteq f(\gamma(U)) \cap f(A) \subseteq \beta(V) \cap f(A)$. This implies that $y \in g C l_{\beta}(f(A))$. Therefore, $f\left(g C l_{\gamma}(A)\right) \subseteq g C l_{\beta}(f(A))$.

2. Let $F$ be any $g$ - $\beta$-closed set of $(Y, \sigma)$. By using (1), we have $f\left(g C l_{\gamma}\left(f^{-1}(F)\right)\right) \subseteq g C l_{\beta}(F)=F$. Therefore, $g C l_{\gamma}\left(f^{-1}(F)\right)=f^{-1}(F)$. Hence $f^{-1}(F)$ is $g$ - $\gamma$-closed set in $(X, \tau)$.

Theorem 19. In 18, the properties of $g-(\gamma, \beta)$-continuity of $f,(1)$ and (2) are equivalent to each other if either the space $(Y, \sigma)$ is $g$ - $\beta$-regular or the operation $\beta$ is g-open.

Proof. It follows from the proof of 18 that we know the following implications: " $g$ - $(\gamma, \beta)$-continuity of $f$ " $\Rightarrow(1) \Rightarrow(2)$. Thus, when the space $(Y, \sigma)$ is $g$ - $\beta$-regular, we prove the implication: (2) $\Rightarrow g$ - $(\gamma, \beta)$-continuity of $f$. Let $x \in X$ and let $V \in \sigma_{g}$ such that $f(x) \in V$. Since $(Y, \sigma)$ is a $g$ - $\beta$-regular space, then by $3, V \in \sigma_{g \beta}$. By using (2) of $18, f^{-1}(V) \in \tau_{g \gamma}$ such that $x \in f^{-1}(V)$. So there exists a $g$-open set $U$ such that $x \in U$ and $\gamma(U) \subseteq f^{-1}(V)$. This implies that $f(\gamma(U)) \subseteq V \subseteq \beta(V)$. Therefore, $f$ is $g$ - $(\gamma, \beta)$-continuous.

Now, when $\beta$ is a $g$-open operation, we show the implication: (2) $\Rightarrow g$ - $(\gamma, \beta)$-continuity of $f$. Let $x \in X$ and let $V \in \sigma_{g}$ such that $f(x) \in V$. Since $\beta$ is a $g$-open operation, then there exists $W \in \sigma_{g \beta}$ such that $f(x) \in W$ and $W \subseteq \beta(V)$. By using (2) of $18, f^{-1}(W) \in \tau_{g \gamma}$ such that $x \in f^{-1}(W)$. So there exists a $g$-open set $U$ such that $x \in U$ and $\gamma(U) \subseteq f^{-1}(W) \subseteq f^{-1}(\beta(V))$. This implies that $f(\gamma(U)) \subseteq \beta(V)$. Hence $f$ is $g-(\gamma, \beta)$-continuous.

Definition 20. A function $f:(X, \tau) \rightarrow(Y, \sigma)$ is said to be 
1. $g$ - $(\gamma, \beta)$-closed if the image of each $g$ - $\gamma$-closed set of $X$ is $g$ - $\beta$-closed in $Y$.

2. $g$ - $\beta$-closed if the image of each $g$-closed set of $X$ is $g$ - $\beta$-closed in $Y$.

Theorem 20. Suppose that a function $f:(X, \tau) \rightarrow(Y, \sigma)$ is both $g$ - $(\gamma, \beta)$-continuous and $g$ - $\beta$-closed,then,

1. For every g $\gamma g$-closed set $A$ of $(X, \tau)$, the image $f(A)$ is $g \beta g$-closed in $(Y, \sigma)$.

2. If $(X, \tau)$ is $T_{\frac{1}{2}}$, then the inverse set $f^{-1}(B)$ is g grg-closed in $(X, \tau)$, for every $g \beta$-closed set $B$ of $(Y, \sigma)$.

Proof. 1 . Let $G$ be any $g$ - $\beta$-open set in $(Y, \sigma)$ such that $f(A) \subseteq G$. Since $f$ is $g$ - $(\gamma, \beta)$-continuous function, then by using 18 (2), $f^{-1}(G)$ is $g$ - $\gamma$-open set in $(X, \tau)$. Since $A$ is $g \gamma g$-closed and $A \subseteq f^{-1}(G)$, we have $g C l_{\gamma}(A) \subseteq f^{-1}(G)$, and hence $f\left(g C l_{\gamma}(A)\right) \subseteq G$. Thus, by $2(1), g C l_{\gamma}(A)$ is $g$-closed set and since $f$ is $g$ - $\beta$-closed, then $f\left(g C l_{\gamma}(A)\right)$ is $g$ - $\beta$-closed set in $Y$. Therefore, $g C l_{\beta}(f(A)) \subseteq g C l_{\beta}\left(f\left(g C l_{\gamma}(A)\right)\right)=f\left(g C l_{\gamma}(A)\right) \subseteq G$. This implies that $f(A)$ is $g \beta g$-closed in $(Y, \sigma)$.

2. Let $H$ be any $g$ - $\gamma$-open set of a $T_{\frac{1}{2}}$ space $(X, \tau)$ such that $f^{-1}(B) \subseteq H$. Let $C=g C l_{\gamma}\left(f^{-1}(B)\right) \cap(X \backslash H)$, then by 7 , $C=g C l_{\gamma}\left(f^{-1}(B)\right) \cap g C l_{\gamma}(X \backslash H)$ and hence by $2(1)$ and $1, C$ is $g$-closed set in $(X, \tau)$. Since $f$ is $g$ - $\beta$-closed function. Then $f(C)$ is $g$ - $\beta$-closed in $(Y, \sigma)$. Since $f$ is $g-(\gamma, \beta)$-continuous function, then by using $18(1)$, we have $f(C)=$ $f\left(g C l_{\gamma}\left(f^{-1}(B)\right)\right) \cap f(X \backslash H) \subseteq g C l_{\beta}(B) \cap f(X \backslash H) \subseteq g C l_{\beta}(B) \cap(Y \backslash B)=g C l_{\beta}(B) \backslash B$. This implies from 9 that $f(C)=$ $\phi$, and hence $C=\phi$. So $g C l_{\gamma}\left(f^{-1}(B)\right) \subseteq H$. Therefore, $f^{-1}(B)$ is $g \gamma g$-closed in $(X, \tau)$.

Theorem 21. Let $f:(X, \tau) \rightarrow(Y, \sigma)$ be an injective, $g-(\gamma, \beta)$-continuous and $g$ - $\beta$-closed function. If $(Y, \sigma)$ is $g$ - $\beta$ - $T_{\frac{1}{2}}$, then $(X, \tau)$ is $g-\gamma-T_{\frac{1}{2}}$.

Proof. Let $G$ be any $g \gamma g$-closed set of $(X, \tau)$. Since $f$ is $g$ - $(\gamma, \beta)$-continuous and $g$ - $\beta$-closed function. Then by 20 (1), $f(G)$ is $g \beta g$-closed in $(Y, \sigma)$. Since $(Y, \sigma)$ is $g-\beta$ - $T_{\frac{1}{2}}$, then $f(G)$ is $g$ - $\beta$-closed in $Y$. Again, since $f$ is $g$ - $(\gamma, \beta)$-continuous, then by $18(2), f^{-1}(f(G))$ is $g$ - $\gamma$-closed in $X$. Hence $G$ is $g$ - $\gamma$-closed in $X$ since $f$ is injective. Therefore, $(X, \tau)$ is a $g-\gamma-T_{\frac{1}{2}}$ space.

Theorem 22. Let a function $f:(X, \tau) \rightarrow(Y, \sigma)$ be surjective, $g$ - $(\gamma, \beta)$-continuous and $g$ - $\beta$-closed. If $(X, \tau)$ is $g-\gamma$ - $T_{\frac{1}{2}}$, then $(Y, \sigma)$ is $g-\beta-T_{\frac{1}{2}}$.

Proof. Let $H$ be a $g \beta g$-closed set of $(Y, \sigma)$. Since $f$ is $g-(\gamma, \beta)$-continuous and $g$ - $\beta$-closed function. Then by 20 (2), $f^{-1}(H)$ is $g \gamma g$-closed in $(X, \tau)$. Since $(X, \tau)$ is $g-\gamma-T_{\frac{1}{2}}$, then we have, $f^{-1}(H)$ is $g$ - $\gamma$-closed set in $X$. Again, since $f$ is $g$ - $\beta$-closed function, then $f\left(f^{-1}(H)\right)$ is $g$ - $\beta$-closed in $Y$. Therefore, $H$ is $g$ - $\beta$-closed in $Y$ since $f$ is surjective. Hence $(Y, \sigma)$ is $g-\beta-T_{\frac{1}{2}}$ space.

Theorem 23. If a function $f:(X, \tau) \rightarrow(Y, \sigma)$ is injective $g-(\gamma, \beta)$-continuous and the space $(Y, \sigma)$ is $g$ - $\beta$ - $T_{2}$, then the space $(X, \tau)$ is $g-\gamma-T_{2}$.

Proof. Let $x_{1}$ and $x_{2}$ be any distinct points of a space $(X, \tau)$. Since $f$ is an injective function and $(Y, \sigma)$ is $g-\beta-T_{2}$. Then there exist two $g$-open sets $U_{1}$ and $U_{2}$ in $Y$ such that $f\left(x_{1}\right) \in U_{1}, f\left(x_{2}\right) \in U_{2}$ and $\beta\left(U_{1}\right) \cap \beta\left(U_{2}\right)=\phi$. Since $f$ is $g-(\gamma, \beta)$ continuous, there exist $g$-open sets $V_{1}$ and $V_{2}$ in $X$ such that $x_{1} \in V_{1}, x_{2} \in V_{2}, f\left(\gamma\left(V_{1}\right)\right) \subseteq \beta\left(U_{1}\right)$ and $f\left(\gamma\left(V_{2}\right)\right) \subseteq \beta\left(U_{2}\right)$. Therefore $\beta\left(U_{1}\right) \cap \beta\left(U_{2}\right)=\phi$. Hence $(X, \tau)$ is $g-\gamma-T_{2}$.

Theorem 24. If a function $f:(X, \tau) \rightarrow(Y, \sigma)$ is injective $g-(\gamma, \beta)$-continuous and the space $(Y, \sigma)$ is $g$ - $\beta$ - $T_{i}$, then the space $(X, \tau)$ is $g-\gamma-T_{i}$ for $i \in\{0,1\}$.

Proof. The proof is similar to 23.

Definition 21. A function $f:(X, \tau) \rightarrow(Y, \sigma)$ is said to be $g$ - $(\gamma, \beta)$-homeomorphism if $f$ is bijective, $g$ - $(\gamma, \beta)$-continuous and $f^{-1}$ is $g$ - $(\beta, \gamma)$-continuous.

Theorem 25. Assume that a function $f:(X, \tau) \rightarrow(Y, \sigma)$ is $g$ - $(\gamma, \beta)$-homeomorphism. If $(X, \tau)$ is $g-\gamma$ - $T_{\frac{1}{2}}$, then $(Y, \sigma)$ is $g-\beta-T_{\frac{1}{2}}$.

Proof. Let $\{y\}$ be any singleton set of $(Y, \sigma)$. Then there exists an element $x$ of $X$ such that $y=f(x)$. So by hypothesis and 14 , we have $\{x\}$ is $g$ - $\gamma$-closed or $g$ - $\gamma$-open set in $X$. By using $18,\{y\}$ is $g$ - $\beta$-closed or $g$ - $\beta$-open set. Hence the space by $14,(Y, \sigma)$ is $g-\beta-T_{\frac{1}{2}}$. 


\section{Functions with $g-\beta$-closed graphs}

For a function $f:(X, \tau) \rightarrow(Y, \sigma)$, the subset $\{(x, f(x)): x \in X\}$ of the product space $(X \times Y, \tau \times \sigma)$ is called the graph of $f$ and is denoted by $G(f)$ [3]. In this section, we further investigate general operator approaches of closed graphs of functions. Let $\lambda:(\tau \times \sigma)_{g} \rightarrow P(X \times Y)$ be an operation on $(\tau \times \sigma)_{g}$.

Definition 22. The graph $G(f)$ of $f:(X, \tau) \rightarrow(Y, \sigma)$ is called $g$ - $\beta$-closed if for each $(x, y) \in(X \times Y) \backslash G(f)$, there exist g-open sets $U \subseteq X$ and $V \subseteq Y$ containing $x$ and $y$, respectively, such that $(U \times \beta(V)) \cap G(f)=\phi$.

The proof of the following lemma follows directly from the above definition.

Lemma 9. A function $f:(X, \tau) \rightarrow(Y, \sigma)$ has $g$ - $\beta$-closed graph if and only if for each $(x, y) \in(X \times Y) \backslash G(f)$, there exist $U \in \tau_{g}$ containing $x$ and $V \in \sigma_{g}$ containing y such that $f(U) \cap \beta(V)=\phi$.

Definition 23. An operation $\lambda:(\tau \times \sigma)_{g} \rightarrow P(X \times Y)$ is said to be g-associated with $\gamma$ and $\beta$ if $\lambda(U \times V)=\gamma(U) \times \beta(V)$ holds for each $U \in \tau_{g}$ and $V \in \sigma_{g}$.

Definition 24. The operation $\lambda:(\tau \times \sigma)_{g} \rightarrow P(X \times Y)$ is said to be g-regular with respect to $\gamma$ and $\beta$ iffor each $(x, y) \in$ $X \times Y$ and each g-open set $W$ containing $(x, y)$, there exist g-open sets $U$ in $X$ and $V$ in $Y$ such that $x \in U, y \in V$ and $\gamma(U) \times \beta(V) \subseteq \lambda(W)$.

Theorem 26. Let $\lambda:(\tau \times \tau)_{g} \rightarrow P(X \times X)$ be a g-associated operation with $\gamma$ and $\gamma$. If $f:(X, \tau) \rightarrow(Y, \sigma)$ is a $g$ - $(\gamma, \beta)$ continuous function and $(Y, \sigma)$ is a $g-\beta-T_{2}$ space, then the set $A=\{(x, y) \in X \times X: f(x)=f(y)\}$ is a g- $\lambda$-closed set of $(X \times X, \tau \times \tau)$.

Proof. We want to prove that $g C l_{\lambda}(A) \subseteq A$. Let $(x, y) \in(X \times X) \backslash A$. Since $(Y, \sigma)$ is $g-\beta-T_{2}$. Then there exist two $g$-open sets $U$ and $V$ in $(Y, \sigma)$ such that $f(x) \in U, f(y) \in V$ and $\beta(U) \cap \beta(V)=\phi$. Moreover, for $U$ and $V$ there exist $g$-open sets $R$ and $S$ in $(X, \tau)$ such that $x \in R, y \in S$ and $f(\gamma(R)) \subseteq \beta(U)$ and $f(\gamma(S)) \subseteq \beta(V)$ since $f$ is $g$ - $(\gamma, \beta)$-continuous. Therefore we have $(x, y) \in \gamma(R) \times \gamma(S)=\lambda(R \times S) \cap A=\phi$ because $R \times S \in(\tau \times \tau)_{g}$. This shows that $(x, y) \notin g C l_{\lambda}(A)$.

Corollary 3. Suppose $\lambda:(\tau \times \tau)_{g} \rightarrow P(X \times X)$ is g-associated operation with $\gamma$ and $\gamma$, and it is g-regular with $\gamma$ and $\gamma$. A space $(X, \tau)$ is $g-\gamma-T_{2}$ if and only if the diagonal set $\triangle=\{(x, x): x \in X\}$ is $g$ - $\lambda$-closed of $(X \times X, \tau \times \tau)$.

Theorem 27. Let $\lambda:(\tau \times \sigma)_{g} \rightarrow P(X \times Y)$ be a g-associated operation with $\gamma$ and $\beta$. If $f:(X, \tau) \rightarrow(Y, \sigma)$ is $g$ - $(\gamma, \beta)$ continuous and $(Y, \sigma)$ is $g$ - $\beta-T_{2}$, then the graph of $f, G(f)=\{(x, f(x)) \in X \times Y\}$ is a g- $\lambda$-closed set of $(X \times Y, \tau \times \sigma)$.

Proof. The proof is similar to 26.

Definition 25. Let $(X, \tau)$ be a topological space and $\gamma$ be an operation on $\tau_{g}$. A subset $S$ of $X$ is said to be g- $\gamma$-compact iffor every g-open cover $\left\{U_{i}, i \in \mathbb{N}\right\}$ of $S$, there exists a finite subfamily $\left\{U_{1}, U_{2}, \ldots, U_{n}\right\}$ such that $S \subseteq \gamma\left(U_{1}\right) \cup \gamma\left(U_{2}\right) \cup \ldots \cup \gamma\left(U_{n}\right)$.

Theorem 28. Suppose that $\gamma$ is g-regular and $\lambda:(\tau \times \sigma)_{g} \rightarrow P(X \times Y)$ is g-regular with respect to $\gamma$ and $\beta$. Let $f:(X, \tau) \rightarrow(Y, \sigma)$ be a function whose graph $G(f)$ is $g$ - $\lambda$-closed in $(X \times Y, \tau \times \sigma)$. If a subset $S$ is $g$ - $\beta$-compact in $(Y, \sigma)$, then $f^{-1}(S)$ is $g$ - $\gamma$-closed in $(X, \tau)$.

Proof. Suppose that $f^{-1}(S)$ is not $g$ - $\gamma$-closed then there exist a point $x$ such that $x \in g C l_{\gamma}\left(f^{-1}(S)\right)$ and $x \notin f^{-1}(S)$. Since $(x, s) \notin G(f)$ and each $s \in S$ and $g C l_{\lambda}(G(f)) \subseteq G(f)$, there exists a $g$-open set $W$ of $(X \times Y, \tau \times \sigma)$ such that $(x, s) \in W$ and $\beta(W) \cap G(f)=\phi$. By $g$-regularity of $\lambda$, for each $s \in S$ we can take two $g$-open sets $U(s)$ and $V(s)$ in $(Y, \sigma)$ such that $x \in U(s), s \in V(s)$ and $\gamma(U(s)) \times \beta(V(s)) \subseteq \lambda(W)$. Then we have $f(\gamma(U(s))) \cap \beta(V(s))=\phi$. Since $\{V(s): s \in S\}$ is $g$-open cover of $S$, then by $g$ - $\gamma$-compactness there exists a finite number $s_{1}, s_{2}, \ldots, s_{n} \in S$ such that $S \subseteq \beta\left(V\left(s_{1}\right)\right) \cup \beta\left(V\left(s_{2}\right)\right) \cup \ldots \cup \beta\left(V\left(s_{n}\right)\right)$. By the $g$-regularity of $\gamma$, there exist a $g$-open set $U$ such that $x \in U, \gamma(U) \subseteq$ $\gamma\left(U\left(s_{1}\right)\right) \cap \gamma\left(U\left(s_{2}\right)\right) \cap \ldots \cap \gamma\left(U\left(s_{n}\right)\right)$. Therefore, we have $\gamma(U) \cap f^{-1}(S) \subseteq U\left(s_{i}\right) \cap f^{-1}\left(\beta\left(V\left(s_{i}\right)\right)\right)=\phi$. This shows that $x \notin g C l_{\gamma}\left(f^{-1}(S)\right)$. This is a contradiction. Therefore, $f^{-1}(S)$ is $g$ - $\gamma$-closed. 
Theorem 29. Suppose that the following condition hold.

1. $\gamma: \tau_{g} \rightarrow P(X)$ is g-open,

2. $\beta: \sigma_{g} \rightarrow P(Y)$ is $g$-regular, and

3. $\lambda:(\tau \times \sigma)_{g} \rightarrow P(X \times Y)$ is associated with $\gamma$ and $\beta$, and $\lambda$ is $g$-regular with respect to $\gamma$ and $\beta$.

Let $f:(X, \tau) \rightarrow(Y, \sigma)$ be a function whose graph $G(f)$ is $g$ - $\lambda$-closed in $(X \times Y, \tau \times \sigma)$. If every cover of $A$ by $g$ - $\gamma$-open sets of $(X, \tau)$ has finite sub cover, then $f(A)$ is $g$ - $\beta$-closed in $(Y, \sigma)$.

Proof. Similar to 28.

\section{Competing interests}

The authors declare that they have no competing interests.

\section{Authors' contributions}

All authors have contributed to all parts of the article. All authors read and approved the final manuscript.

\section{References}

[1] T.V. An, D.X. Cuong and H. Maki, On operation-preopen sets in topological spaces, Scientiae Mathematicae Japonicae Online, 68 1 (2008), 11-30, (e-2008), 241-260.

[2] C. Carpintero, N. Rajesh and E. Rosas, Operation approaches on b-open sets and applications, Bulletin of Parana's Mathematical Society, 301 (2012), 21-33.

[3] T. Husain, Topology and Maps, Plenum press, New York, (1977).

[4] D. S. Jankovic, On functions with $\alpha$-closed graphs, Glasnik Matematicki, 1838 (1983), 141-148.

[5] N. Levine, Generalized closed sets in topology, Rendiconti del Circolo Matematico di Palermo, 19 l (1970), 89-96.

[6] S. Kasahara, Operation compact spaces, Math. Japonica, 24 l (1979), 97-105.

[7] G. S. S. Krishnan, M. Ganster and K. Balachandran, Operation approaches on semi-open sets and applications, Kochi Journal of Mathematics, 2 (2007), 21-33.

[8] H. Ogata, Operation on topological spaces and associated topology, Math. Japonica, 361 (1991), 175-184.

[9] S. Tahiliani, Operation approach to $\beta$-open sets and applications, Mathematical Communications, 16 (2011), 577-591. 